\title{
Modelos de Integración de la Gestión de la Cadena de Suministro en el Sector de la Construcción
}

\author{
Integration Models for Supply Chain Management in the Construction \\ Sector
}

\author{
J. Francisco Carbonell Ureña \\ Dpto. Organización de Empresas. Universidad Politécnica de Valencia. \\ Camino de Vera s/n , 46022. Valencia. \\ Francisco.Carbonell@iese.net
}

Fecha de recepción: 28-05-2010

Fecha de aceptación: 19-10-2010

Resumen: Actualmente el sector de la construcción está experimentando grandes cambios en todos los aspectos relacionados con su negocio. Ahora más que nunca, cuando la crisis mundial obliga a la utilización de sistemas de gestión más eficientes, se requieren de modelos integrales de gestión de las cadenas de suministro del sector de la construcción. Este artículo analiza modelos existentes de integración de la Cadena de Suministro y propone el Modelo de Organización Global (GOM) caracterizado por la integración de las cadenas productivas de las empresas participantes en la cadena de suministro con el cliente como eje principal y vehículo integrador de todo el proceso.

Palabras clave: construcción, modelos integrales de gestión, cadena de suministro, cadenas virtuales, modelo de organización global.

Abstract: Currently the construction industry is undergoing great changes in all aspects of the business. Now more than ever, when the global crisis requires the use of more efficient management systems, will require comprehensive models for managing supply chains in the construction sector. This article examines existing models of integration of supply chain and proposes the Global Organization Model (GOM) characterized by the integration of productive chains of the companies participating in the supply chain with the customer as the main shaft and all inclusive vehicle the process.

Key words: construction, comprehensive management models, supply chain, virtual chains, global organization model.

\section{Introducción}

La cadena de suministro en el sector de la construcción es posiblemente uno de los sistemas logísticos y productivos de mayor antigüedad y con un desarrollo e implantación más extensa a lo largo de todo nuestro planeta. El sector de la construcción está pasando actualmente por procesos de internacionalización y/o especialización de sus unidades productivas, debiendo ser su tendencia la de convertir sus cadenas productivas en auténticas «cadenas virtuales», en las que se incluyan los proveedores y subcontratas como parte de las mismas (Vicedo et al., 2005)

En primer lugar cabe señalar que el sector de la construcción se caracterizó, en la década de los ochenta y los noventa por: la perdida de valor comercial de la propiedad raíz, la confusión en la me- dición de la creación arquitectónica, con una visión economista y competitiva que preside toda actividad productiva; y el abandono del sector y de las universidades formadoras de profesionales, de los objetivos sociales y culturales para impulsar su desarrollo (Vélez Moreno y Ligia María, 200 I).

El sector de la construcción en nuestros días se ha caracterizado por la estacionalidad, baja especialización de las actividades, atomización financiera y organización piramidal vertical de las empresas.

La estacionalidad ocasionada por la subcontratación de servicios y mano de obra, y el subarriendo de equipos, delimita la flexibilidad y eficiencia en el control de los procesos dentro de la empresa.

La poca especialización de las actividades y tareas propias de la ejecución de las obras, producto de la 
baja cualificación de la mano de obra y de los bajos salarios.

La organización del personal en las empresas es piramidal vertical, no son organizaciones matriciales que flexibilicen la toma de decisiones y haga controles paralelos en los diferentes frentes de trabajo, (Vélez Moreno y Ligia María, 200 I).

En la actividad de construcción se presentan características propias que la diferencian notablemente de otros sectores, especialmente de la industria manufacturera. La naturaleza del producto final constituye la característica distintiva del sector. Ésta viene dada por su unicidad, inmovilidad y variedad (Gonzalez et al., 2000).

a) Unicidad. Los productos de construcción son elaborados por proyecto, adaptándose al promotor, a su emplazamiento y a su funcionalidad. Esta unicidad de los proyectos desaconseja el uso de medios de producción especializados para cada proyecto, a diferencia de aquellas industrias que elaboran productos en serie. No obstante, en algunas actividades intermedias existe cierto grado de estandarización que facilita el aprovechamiento de las ventajas de especialización (prefabricación de vigas, paramentos, puertas, ventanas, etc.) .

b) Inmovilidad. La construcción se caracteriza por el levantamiento de una o varias estructuras inmóviles en un lugar predeterminado en el que se efectúan buena parte de las transformaciones. Por lo tanto, son los recursos productivos los que se desplazan hacia los productos en curso, y no éstos hacia los activos, tal y como suele ocurrir en la industria manufacturera. La consecuencia de esta ubicación exclusiva es que cada nuevo proyecto requiere un nuevo centro de trabajo, al no ser posible concentrar la actividad productiva en un único lugar.

c) Variedad. La tercera cualidad distintiva de la construcción es la elaboración de una gran variedad de productos finales, que incluye desde pequeñas reparaciones domésticas hasta grandes obras de infraestructura. Cada tipo de producto final incorpora su propia combinación de actividades intermedias heterogéneas. En consecuencia, a medida que una empresa integra horizontalmente tipos adicionales de construcción, ha de acometer actividades intermedias más dispares. Esto provoca que las actividades que realizan las empresas constructoras no sólo sean heterogéneas por su ubicación geográfica, sino también por su naturaleza.

Estas propiedades, relacionadas básicamente con la tecnología física, alteran algunas de las características de las transacciones ya comentadas y, en consecuencia, el tipo de mecanismos que los participantes deben introducir para salvaguardar sus intercambios.

En segundo lugar, y analizando las empresas del sector vemos que la industria de la construcción se puede caracterizar por empresas que manejan muchos proyectos en diferentes zonas geográficas al mismo tiempo y que a su vez tienen invertidas grandes cantidades de dinero en dichos proyectos (Rey Román et al., 2005). Este cambio geográfico es uno de los principales factores que hace de los diferentes proyectos de construcción tanto inmobiliaria como de infraestructura, únicos e irrepetibles. Nunca se tendrán dos proyectos iguales al cien por cien.

En la actualidad el sector de la construcción se caracteriza por ser de una naturaleza cambiante, cada segundo que transcurre implica nuevos retos y la problemática de estar preparados para enfrentarlos (Pereira y Chaparro, 2004). Esta situación obliga a las empresas a asumir una posición diferente que no necesariamente debe ser la de anticipar el futuro, sino adaptarse rápidamente a los cambios del presente, y estar preparados para el futuro, a fin de cuentas éste último depende en gran medida de lo que ocurre en el presente.

Ante esta situación, es de esperar que se cuente con un sistema organizacional capaz de aprender a adaptarse al entorno que rodea a la empresa. Dentro del mismo, la gestión empresarial representa una parte fundamental, que a nuestro juicio requiere de soluciones imaginativas que permitan a las empresas buscar soluciones competitivas dentro del mercado que en cada momento se encuentre.

En tercer lugar, y dado lo comentado anteriormente los modelos de integración de la cadena de suministro propuestos por diferentes autores a lo largo de las últimas décadas presentan distintos enfoques de la problemática a resolver, así como diferentes soluciones en cuanto a la estructura del modelo y las fases de aplicación. Estos modelos nos pueden ayudar a enfocar el trabajo en las empresas y abordar soluciones que mejoren el funcionamiento de las mismas. 
Así mismo dichas características configuran un sector que presenta una alternativa interesante, a nuestro juicio, para desarrollar modelos de integración que permitan a las empresas que participan en el mismo desarrollar ventajas competitivas de forma conjunta y animando a la integración de sus cadenas de suministro.

Este artículo se organiza del siguiente modo: en la sección 2 se analizan los diferentes modelos de integración de la cadena de suministro existentes; en la sección 3 se propone el Modelo de Organización Global (GOM); en la sección 4 se detallan las conclusiones de esta investigación.

\section{Modelos de integración de la Cadena de Suministro}

\section{I. Un Repaso al Estado del arte}

SridharTayur et al. (1998) recogen en su libro Quantitative Models for Supply Chain Management un resumen de los modelos cuantitativos destinados a la mejora de la Gestión de La Cadena de Suministro. Stadtler y Kilger (2008) recogen con énfasis los modelos para la gestión de la cadena de suministro y su implementación en distintas empresas. Así mismo es interesante el trabajo de Narasimhan R. y Santosh Mahapatra (2004) en dónde los autores reconocen la dificultad de realizar una tipología de modelos de decisión para la integración global de la Cadena de Suministro y recogen en una tabla aquellos artículos relacionados con aspectos concretos de la Cadena de Suministro. En este mismo trabajo, Narasimhan R. y Santosh Mahapatra (2004), los autores recogen cinco ejemplos de modelos de decisión en la gestión de la cadena de suministro que ilustran y demuestran la importancia que la integración de las decisiones tiene a través de la cadena de suministro.

\subsection{Modelos de Integración de la Cadena de Suministro}

Para el propósito que perseguimos en este artículo que es la presentación de un nuevo modelo se cree más interesante realizar en primer lugar una revisión de los modelos de Integración en la gestión de la Cadena de Suministro que se puede empezar con Stevens (1989) quién propuso un modelo de integración con cuatro fases: integración básica, integración funcional interna, integración del suminis- tro y la demanda de las propias compañías a lo largo de la cadena, y la integración total de la cadena de suministro.

Hewitt (1994) extendió el modelo de Stevens a una quinta fase que se podría dedicar a una mejor administración y re-diseño de los procesos de negocio globales, buscando mejorar la eficiencia y la eficacia globales de tales procesos.

Scott y Westbrook (1991) sugirieron un modelo de tres fases para obtener una cadena de suministro integrada: I) la «fase de estudio», donde se analizan los lead times y los niveles de inventario para ver mejoras potenciales, 2) una «fase de posicionamiento», para identificar nuevas oportunidades que surgen como consecuencia de las actividades de colaboración entre los miembros de la cadena, y 3) una «fase de acción» para llevar a cabo los planes anteriores.

Towill et al. (1992) presentaron un enfoque de integración, similar al de Stevens, y se basaron en principios de dirección de operaciones para reducir la amplificación de la señal de la demanda a lo largo de la cadena.

Bowerbox (1997) plantea la idea de dos tipos de integración: interna y externa, y sugiere que la creación de beneficios de tiempo y ubicación en la cadena, exige compartir la información permitiendo acuerdos comerciales convenientes con ese propósito, y también exige la existencia de un ambiente apropiado para las transacciones financieras.

\subsection{Modelos de Integración en el sector de la construcción}

Además de los modelos de integración genéricos existen también modelos de integración relacionados con el sector de la construcción. En el presente artículo se verán con más detalle tres modelos que recogen aspectos fundamentales relacionados con el modelo que se propone. Estos modelos son Crane et al. (1997), los propuestos por Love et al. (2004), y Vrijhoef y Ruben (2007).

\subsubsection{Partnerig Process Model, T. G. Crane y et al. (1997)}

Uno de los aspectos fundamentales cuando hablamos de la integración de la cadena de suministro 
son las relaciones entre el cliente y el proveedor. En este aspecto es interesante revisar un modelo para la creación de relaciones de partenariado. Estas relaciones se pueden entender como uno de los caminos para llegar a integrar procesos dentro de la Gestión de la Cadena de Suministro, es en esta medida que se cree de interés ver el detalle de este modelo por su aportación a la idea de la integración de la Cadena de Suministro que se está exponiendo en el presente artículo. En los últimos las relaciones de alianzas se han ido incrementando paulatinamente en empresas de ingeniería y construcción. Asimismo muchas investigaciones se han realizado para describir el concepto de partenariado, y como se debe estructurar los grupos de trabajo y los be- neficios del mismo (Abudayyeh 1994; Brown 1993; Crane 1995; Liska 1993; Poirier and Houser 1993). Éstas publicaciones han demostrado que las relaciones de partenariado creadas en entornos armoniosos han facilitado la reducción de costes y litigios legales, han incrementado la calidad productividad y beneficios. Existen trabajos que han hablado de cómo crear esas relaciones de partenariado, Shaokai y Hong (2006) presentan un modelo para evaluar la aplicabilidad de este tipo de relaciones en empresas del sector de la construcción.

Crane et al. ( 1997) nos proponen un proceso para realizar una alianza con éxito. Los autores basan su modelo en cinco fases que según indicamos en la Fi-

Figura 3

Partnerig Process Model, G. Crane y et al. (1997)

\section{Objetivos:}

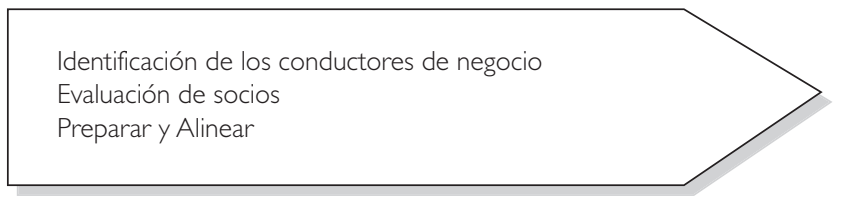

Objetivos:

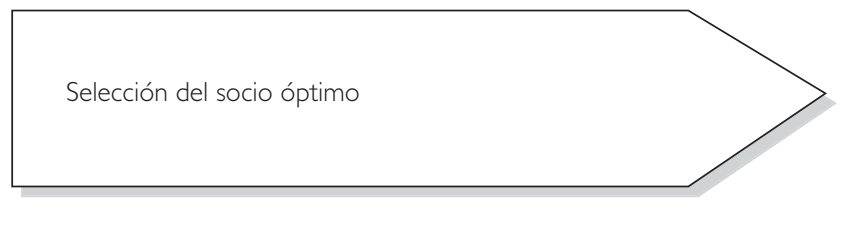

Objetivos:

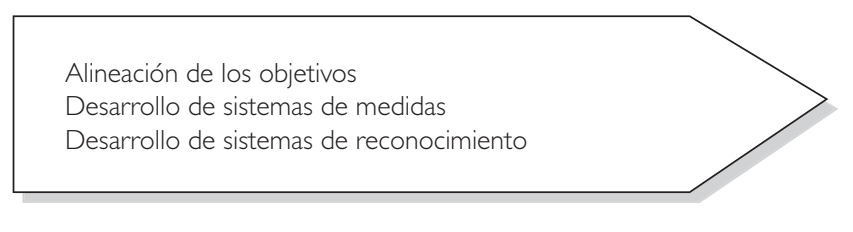

Objetivos:
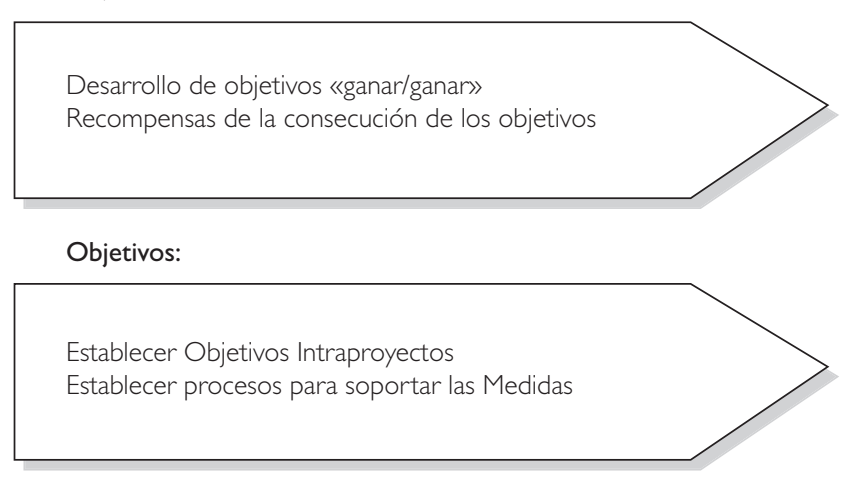

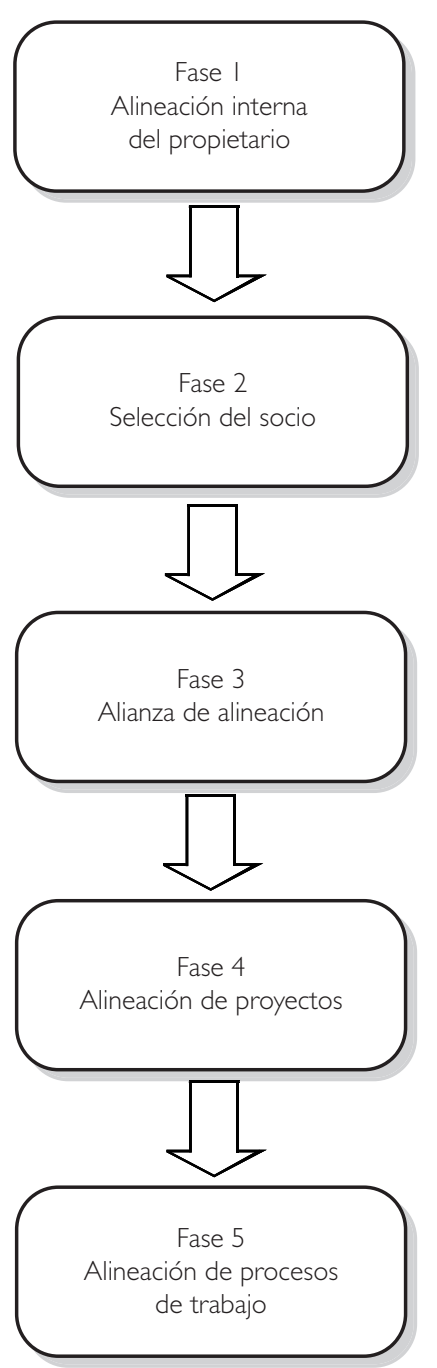


gura 3 Partnerig Process Model,T. G. Crane y et al. (1997), van desde la alineación interna de la empresa hasta la alineación del proceso de trabajo.

Cada una de estas fases tiene sus objetivos y sus factores críticos pero muestran una hoja de ruta que permite desarrollar el modelo. Los factores críticos se pueden ver en el estudio citado así como los detalles de cada una de las fases expuestas aquí.

\subsubsection{Seamless supply chain management model for construction. Love et al. (2004)}

Viendo con más detalle lo que los autores llaman «Seamless supply chain management model for construction» propuesto por Love et al. (2004) este modelo de integración, se basa en un núcleo donde un equipo multidisciplinar de diseño trabaja de forma colaborativa en el aprendizaje y desarrollo del producto. La novedad aportada por este trabajo es que desarrolla un equipo que pilota la integración de la cadena de suministro y permite su integración. Otro modelo de interés para el modelo que se presenta es una propuesta de sistematización para la creación de relaciones de alianza entre compañías, «Partnering Process Model» propuesto por Crane et al. (1997). En el se verá como crear un modelo para la constitución y desarrollo de las relaciones de alianza entre compañías. Por último se verá los trabajos realizados porVrijhoef Ruben (2007) y los modelos de integración propuestos para la cadena de suministro en el sector de la construcción.

Como recoge Bygballe L. et al. (2007) La Gestión de la Cadena de Suministro es un tópico «caliente» en el sector de la construcción, tanto para las empresas como para el mundo científico (ver Akintoye et al 2000, Love et al. 2004, Proverbs and Holt 2000 y Voordijk et al. 2000). Esto a pesar de que la industria es considerada como una tipología distinta a otras industrias como puede ser la industria de automóviles cuya gestión de la cadena de suministro está mucho más desarrollada (Vrijhoef y Koskela 2000, Ballard y Howell 2003, Winch 2003).

La industria de la construcción está altamente fragmentada y esto está en el origen de los críticos y pobres resultados que se obtienen, así como en la baja innovación de los procesos (Love et al., 2002). Para mejorar los resultados, particularmente las relaciones inter-organizacionales, las organizaciones necesitan considerar la formación de alianzas con sus principales proveedores. Algunas empresas de construcción han empezado a establecer alianzas de corto plazo con sus clientes y proveedores como parte de una estrategia de gestión de la cadena de suministro. Sin embargo, estas alianzas de corto plazo no proporcionan el feed-back necesario. Es necesario desarrollar una cooperación, confianza y desarrollo del conocimiento a largo plazo. Es por esto que Love et al (2002) nos proponen la necesidad que tienen las organizaciones de desarrollar alianzas a largo plazo, como parte necesaria del desarrollo del conocimiento en el sector. Las implicaciones de formar diferentes tipos de estrategias para alianzas en la construcción han sido muy discutidas. El modelo inter-organizacional que ellos proponen puede ser usado para ir desarrollando el aprendizaje y está basado en las principales reglas del TQM.

El modelo para la construcción de alianzas basado enTQM y que integra la cadena de suministro lo podemos ver en la Figura I Modelo de alianzas para la construcción de Love P.E.D. et . (2002).

El paso innovador que este modelo presenta es el concepto de lo que los autores Ilaman Systems Thinking. Como se puede ver en el modelo de la figura los componentes esenciales incluyen: cultura de aprendizaje, conocimiento y comunicación, cambios en los modelos mentales, estructura y procesos de aprendizaje y desarrollo de relaciones de aprendizaje. Este es un modelo que está inspirado en la estructura de aprendizaje propuesta por Morrison y Mezentseff (1997) pero adaptado a la estrategia de las empresas de construcción.

Lo que se conoce como «seamless supply chain management model for construction» propuesto por P.E.D. Love et al., 2004 habla de un modelo de integración para la gestión de la cadena de suministro de la construcción. Los citados autores comentan que iniciativas como ésta son a menudo usadas en conjunción con las prácticas tradicionales de dirección y control de proyectos en la cadena de suministro dando como resultado una sensación de mejora que está limitada a nivel de su proceso (Vrijhoef y Koskela, 2000). Más todavía, procesos basados en el T.Q.M. no han sido puestos en práctica como filosofía en las organizaciones de construcción, quizá porque muchas compañías no tienen las habilidades necesarias y experiencia requerida para utilizar de forma efectiva las herramientas y técnicas necesitadas para mejorar la cadena de suministro de la cons- 
Figura 1

Modelo de alianzas para la construcción de Love P.E.D. et al (2002)

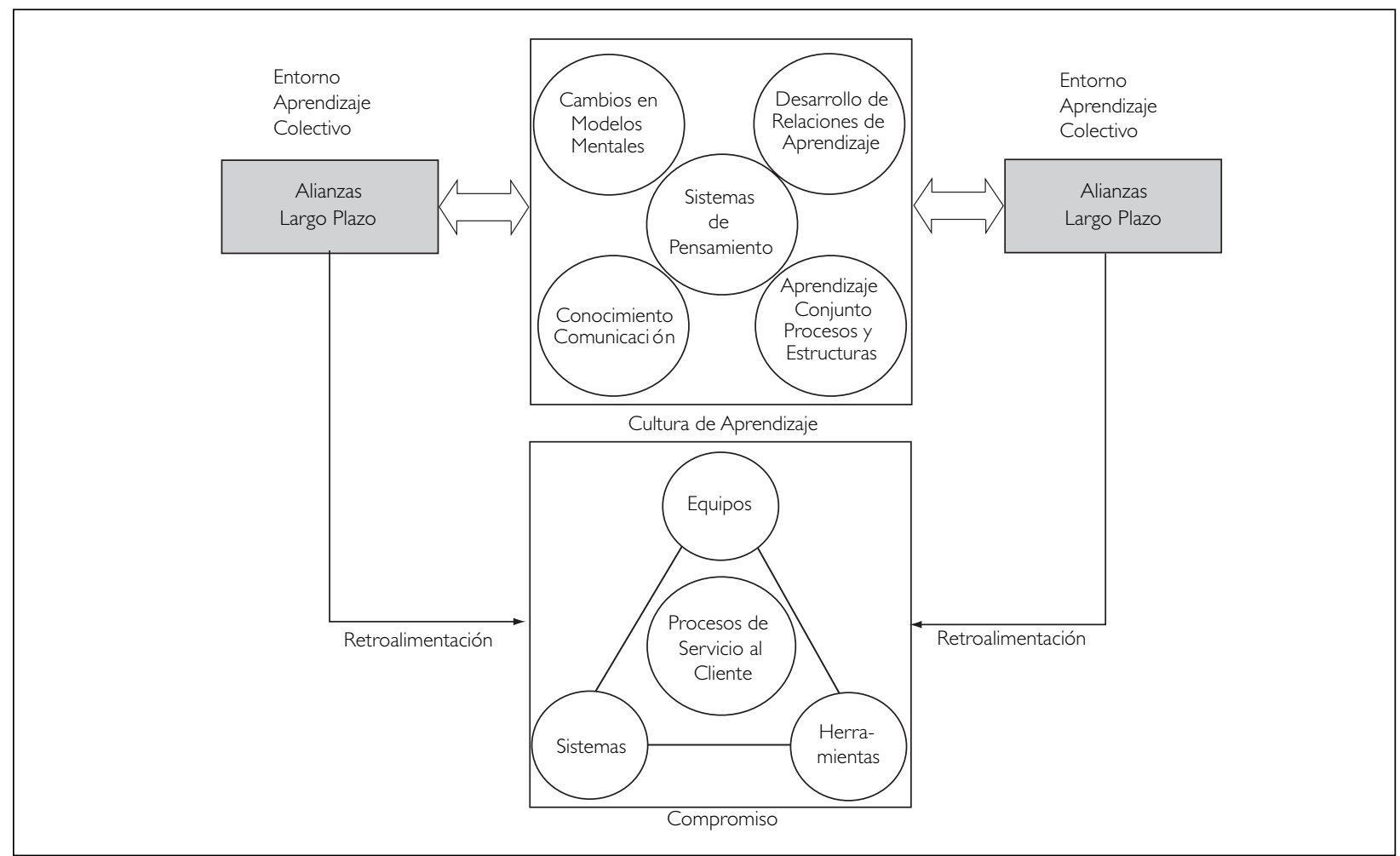

trucción (Love y Sohal, 2002). Más que aplicar iniciativas específicas en la gestión de la cadena de suministro de forma fragmentada y orientada a sosegar problemas, el propósito de este modelo es realizar un acercamiento a las necesidades y soluciones de la gestión de la cadena de suministro de la construcción de forma global. Con este modelo se pretende integrar diseño y procesos constructivos y presentar un modelo para su discusión.

P.E.D.Love et al. 2004 mantienen en su modelo que la separación del diseño y los procesos de producción en proyectos han sido altamente criticados durante los últimos 50 años (ejemplo: Simon Report, 1944; Banwell 1964; Latham 1994; y Egan 1998). Consecuentemente, puede aparecer finalmente lo que llamamos un puente para superar este «gap» creando el modelo que los autores proponen donde la relación cara a cara entre las varias fases del proyecto se integra de forma circular de una forma única. Love et al., 2002, esta organización involucrada con el proyecto es a la vez cliente y proveedor, y debe considerarse de ambas maneras, de esta forma pueden ir dando y creando valor a través de la cadena de suministro, ver Figura 2 Interface de relación cliente proveedor en un proyecto de Cade- na de Suministro P.E.D. Love et al. (2004). En el fondo, y de acuerdo con lo expresado por Lamming, 1996, el valor debe darse más por las personas en la atención en el proceso que porque sea un coste.

Para mejorar la comunicación y minimizar las barreras a los flujos de información en los proyectos, Love et al., 1998, proponen que la tradicional forma de estructurar los proyectos, usada en proyectos de procuración, debe ser reemplazada por una estructura horizontal basada en un equipo multidisciplinar. Esto implica que los individuos y los grupos trabajan juntos de forma continuada en vez de secuencialmente, para diseñar y desarrollar los productos y los procesos, así como para identificar o innovar en los materiales y equipos necesarios para el proceso productivo.

\subsubsection{Supply chain integration for achieving best value for construction clients: client-driven versus supplierdriven integration. Vrijhoef y Ridder (2005)}

Por último, en este apartado, y dado que los procesos de diseño y construcción tienen una importan- 
Figura 2

Interface de relación cliente proveedor en un proyecto de Cadena de Suministro P.E.D. Love et al. (2004)

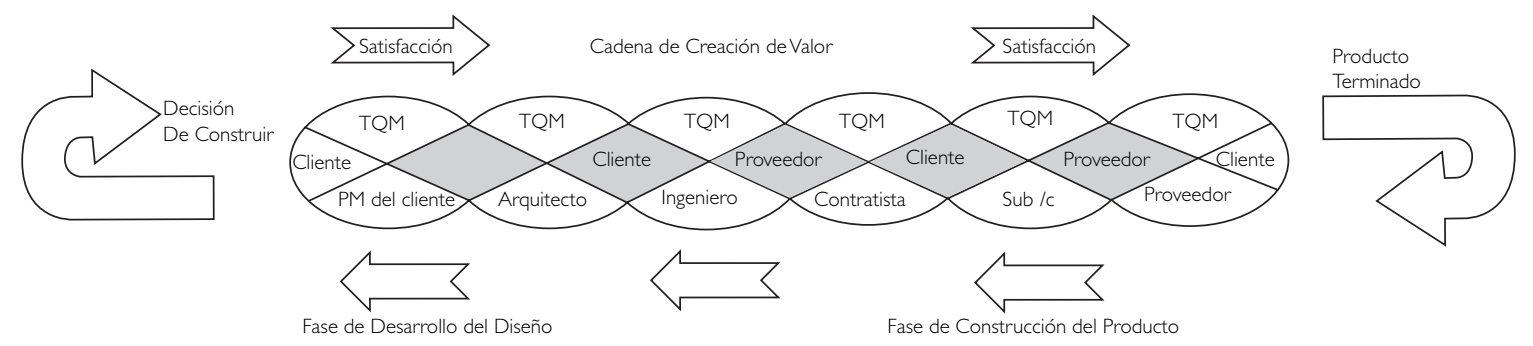

cia vital en el resultado que se va a obtener del proceso global del que se está hablando, se verá un modelo de integración de la cadena de suministro del sector de la construcción propuesto por Vrijhoef y Ridder (2005), en el cuál este concepto se desarrolla de forma explícita. Para alcanzar esto, todas las empresas y funciones deberían estar conectadas, operando como si fueran una fabrica sin muros. Fawcett y Magnan (2002) argumentan que a menudo la integración de la cadena de suministro es una tarea difícilmente alcanzable en su totalidad.

En la construcción, la demanda y la Cadena de Suministro no están a menudo, bien integradas (ver Figura 4: Demanda y Cadena de Suministro en la construcción Vrijhoef y Ridder (2005)). En definitiva parece lógico debido a que en la práctica la integración de los sistemas debe ser costosa y compleja, sobre todo en una industria que debe organizarse principalmente en proyectos. Por otra parte, aparece la necesidad de integración de ambas partes, la demanda y la cadena de suministro. Vrijhoef (2007) propone la aparición de dos nuevos roles, un sistema integrador de la demanda y un sistema integrador de la cadena de suministro. Figura 5. El papel del integrador de la demanda y la cadena de suministro. Vrijhoef y De Ridder (2005).

La propuesta de Ruben Vrijhoef (2007) con los sistemas de reingeniería e integración es mejorar la funcionalidad de la cadena de suministro de la construcción. Esto incluye un cambio de procesos, y de modelo, lo que llama una reconstrucción organizacional de la cadena de suministro. Para alcanzar esto, primero de todo la cadena debe ser descompuesta. Seguidamente debe reorientarse y reconfigurarse las funciones y los interfaces entre las mismas. Haciendo esto los problemas endémicos y las irracionalidades debe resolverse, eliminando las existentes ineficiencias. El efecto se conseguirá controlando las diferentes funciones con una probablemente mayor centralización en una sola entidad, una empresa extendida.

Figura 4

Demanda y cadena de suministro en la construcción. Vrihoef y De Ridder (2005)

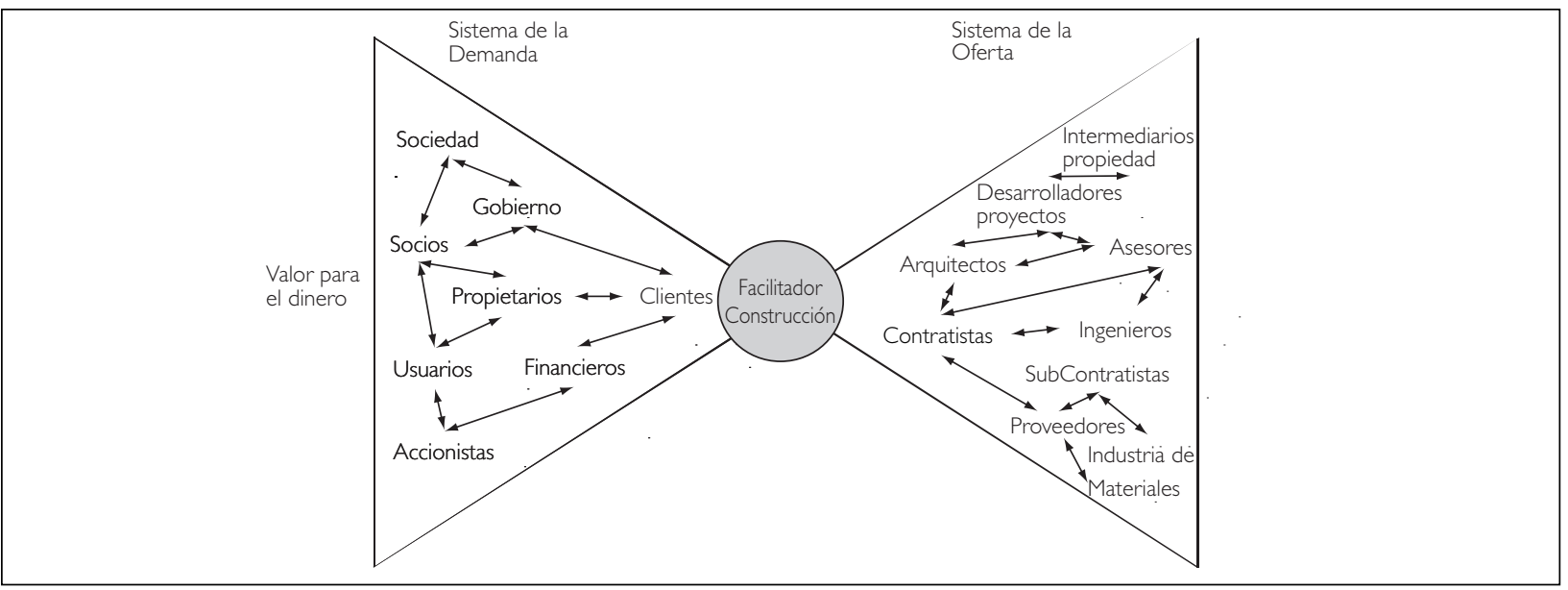


Esta reorganización de la demanda y la integración de la cadena propuesta por Ruben Vrijhoef (2007) se basa en dos actores de la cadena que son el integrador de la demanda y el integrador de la cadena de suministro, Vrijhoef y De Ridder (2005), ver Figura 6.

Figura 5

El papel del integrador de la demanda y la cadena de suministro. Vrijhoef y De Ridder (2005).

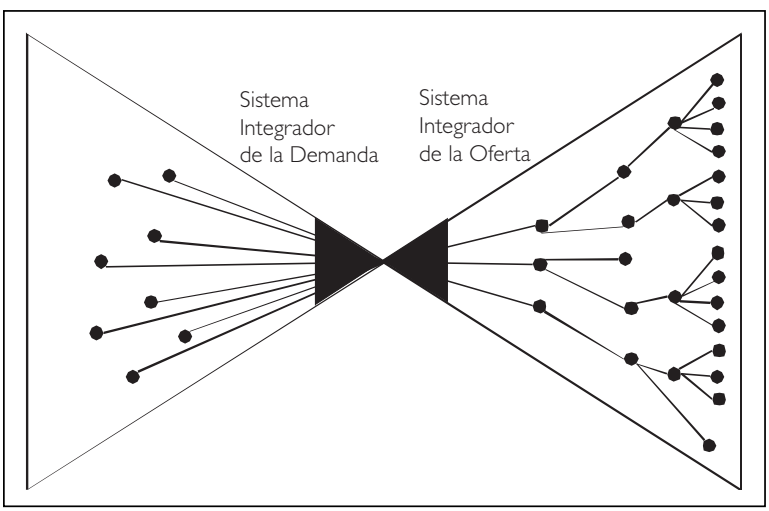

Con esto se pretende mejorar los resultados de la industria de la construcción a través del incremento de la eficiencia y la efectividad integrando los procesos a través de la Cadena de Suministro. Como resultado se obtendrá una mejora económica de los resultados del sector, una mejora de la satisfacción de clientes y usuarios, una reducción de la utilización de capital, los humanos y los recursos naturales empleados en los proyectos, así como una mejora de la salud y el bienestar global de la sociedad.

\section{Propuesta de un modelo de integración para la cadena de suministro en el sector de la construcción: Modelo de Organización Global (GOM)}

El Modelo de Organización Global es un desarrollo conceptual, que ayuda a la implantación práctica de una filosofía que se pretende trasladar en el desarrollo de una estrategia diferente de integración de la cadena de suministro de la construcción. Esta filosofía se basa en la figura del cliente y su fidelización como eje principal de desarrollo del modelo propuesto. En este apartado se va a contemplar el funcionamiento general del modelo que se presenta basado sobre todo en la creación, personalización, desarrollo, diseño y construcción de productos finales. Un concepto parecido al que se expondrá a continuación se ha visto en Love et al. (2004). Los autores citados configuran un modelo de funcionamiento para la gestión de la Cadena de Suministro de la Construcción creando un marco de funcionamiento en un ambiente abierto, con una colaboración y un enfoque común desde el prisma de lo que ellos llaman un colectivo multidisciplinar que va creando a su vez un colectivo de aprendizaje y conocimiento.

La integración consiste, de alguna manera, en facilitar los flujos de materiales, información, decisiones y control a través de la organización ligando las funciones con la información, los recursos, las aplicaciones y las personas, con la finalidad de mejorar la comunicación, la cooperación y la coordinación en la empresa, de tal forma que ésta se comporte como un todo, y que funcione alineada con la estrategia de la empresa.

Papeles del Integrador de la demanda e del integrador de la cadena de suministro Vrijhoef y De Ridder (2005)

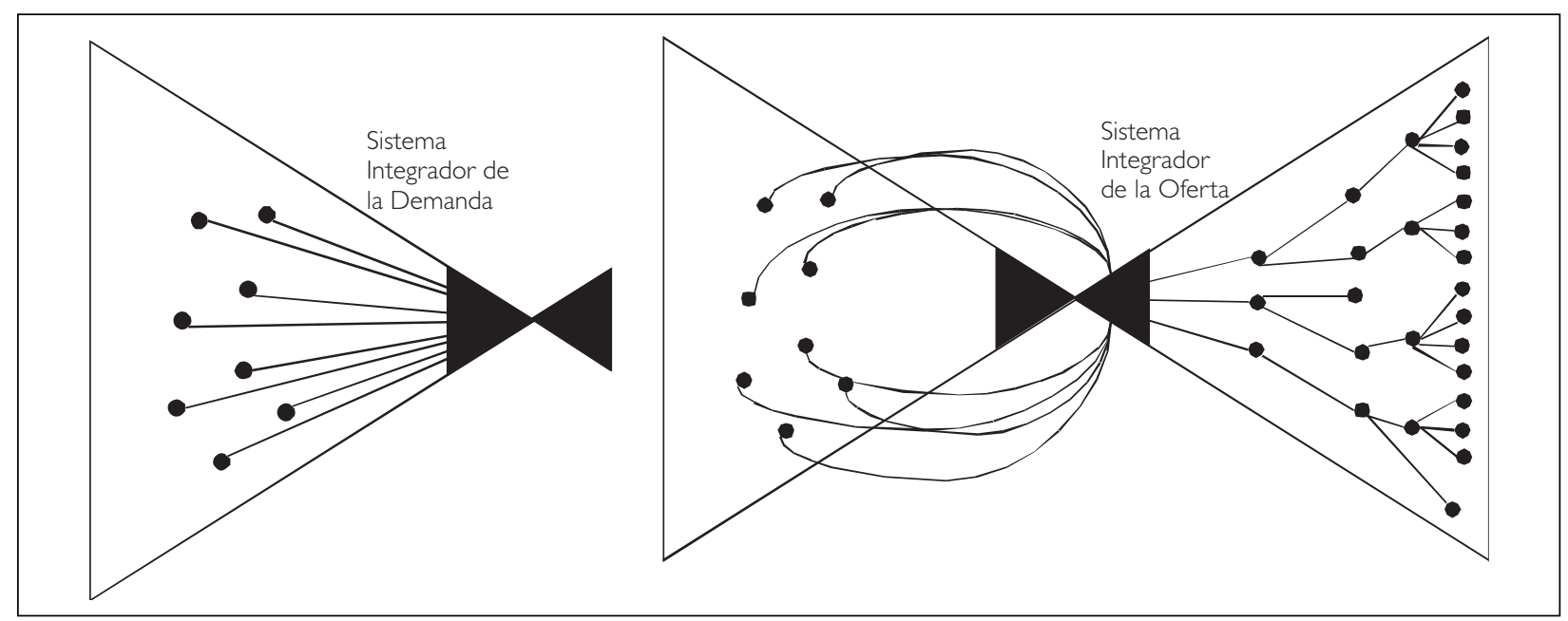


El primer factor distintivo en la propuesta que se presenta es la influencia y el papel del cliente en todo el funcionamiento del modelo. El papel que la integración del cliente tiene en el modelo enriquece los conceptos anteriores y da una visión novedosa de la manera de entender las relaciones dentro de la gestión de la Cadena de Suministro en el sector de la construcción.

Para poder describir de una mejor forma el concepto propuesto se dispone de la Figura 7. En esta representación gráfica se puede ver, si se observa desde el centro hacia fuera, un primer círculo que se ha llamado información directa del cliente, es la que se debe obtener del contacto con el destinatario final del proceso productivo. El Funcionamiento del cliente se entiende como gestión del conocimiento y se basa en una relación continua con él en todo el proceso de diseño, desarrollo y creación del producto. Esta relación continua con el cliente se fundamenta en un equipo multidisciplinar de diseño del producto en donde aparecen figuras como los arquitectos, interioristas, constructores, técnicos, estructuristas, etc. Este equipo tiene como objetivo fundamental garantizar que el producto es el que el cliente desea y es, a su vez, el que se va a obtener.

Figura 7

Funcionamiento general: Modelo de Organización Global (GOM). Elaboración propia

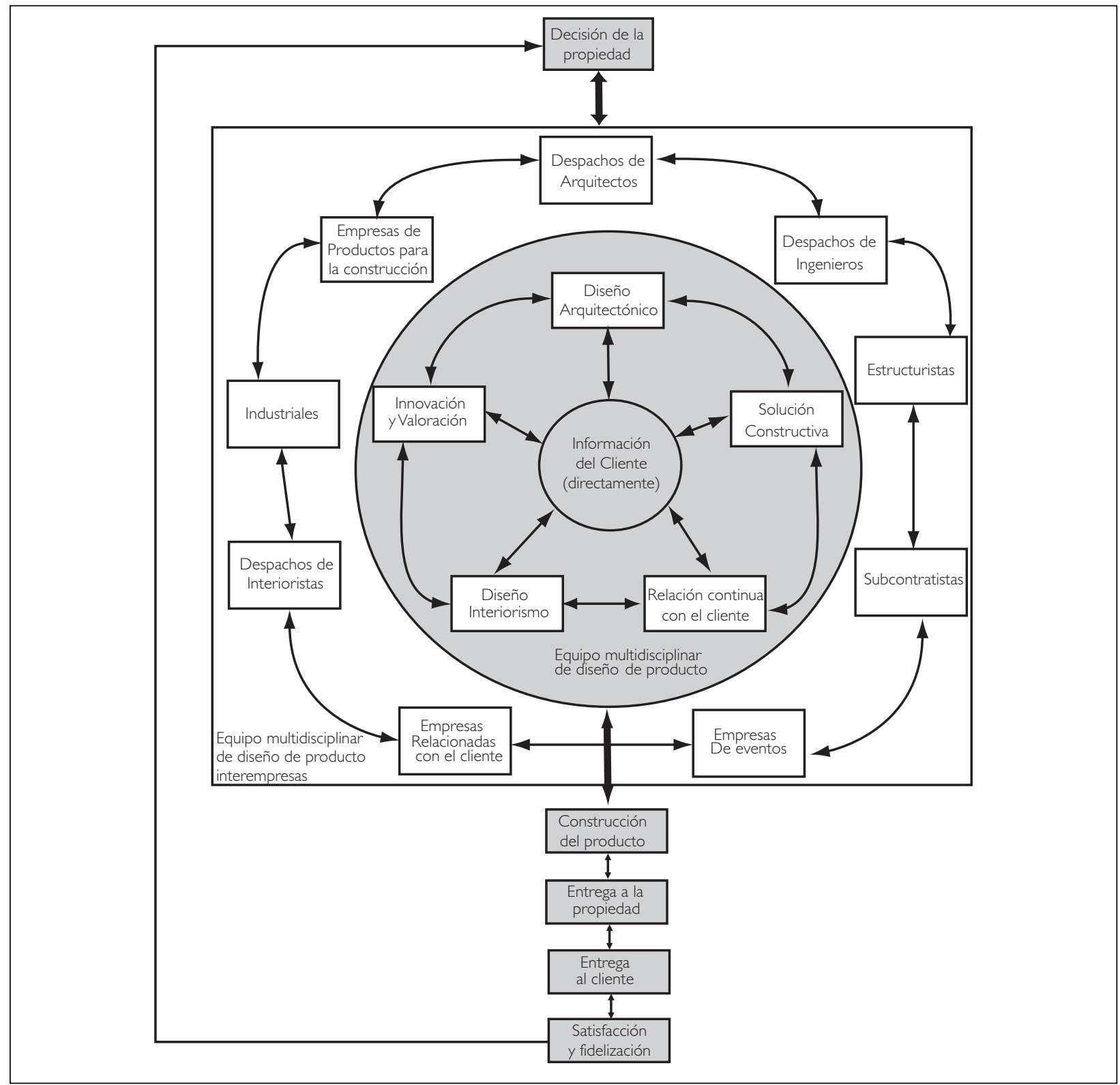


Así como en el modelo de Love et al (2004) existía un equipo multidisciplinar que guiaba el proceso de forma que sumía el papel de integrador de la Cadena de Suministro que propone Vrijhoef y De Ridder (2005) en su modelo, el cliente y lo que hemos llamado el círculo del cliente son en el modelo propuesto los que dirigen e impulsan la integración de la Cadena de Suministro. De esta manera no solo conseguimos la integración de la Cadena que proponen los autores citados con anterioridad sino que a su vez alineamos los resultados del proceso productivo con la voluntad del cliente y sus necesidades. Las empresas que se ven involucradas en este proceso y que forman cada cadena de valor deberán de concentrarse en lo que realmente saben hacer mejor que ningún otro competidor, y además aprender a cooperar con terceros en la generación de valor, en una base de confianza mutua. Para que esta cadena de valor tenga como punto de orientación básico el cliente, buscando responder a sus necesidades, deseos y exigencias, se hará necesario un nuevo sistema organizativo, capaz de adaptarse a los cambios e incluso de adelantarse a los mismos, aprovechando las oportunidades de negocio que puedan presentarse (Vicedo et al. 2009).

El Círculo del cliente que como se ha dicho es sobre lo que pivota el equipo multidisciplinar consiste en un trabajo continuo y circular sobre el eje del cliente. Una primera parte más externa de este doble círculo está relacionada con el cliente. Su captación, fidelización, la gestión del conocimiento y de la información que se tiene del cliente, la personalización del producto, diseño del mismo, creación, entrega, servicio postventa y satisfacción del cliente, son aspectos que sólo podemos realizar con el cliente. Sin el cliente toda esta parte no tiene sentido. Una parte más interna de este modelo es la relación con los proveedores (técnicos y no técnicos), con los empleados de las distintas empresas que integran la Cadena de Suministro y la relación con los accionistas de estas mismas empresas.

El equipo multidisciplinar de diseño es el núcleo central de la propuesta del modelo de integración para la gestión de la cadena de suministro en el sector de la construcción. Una definición adecuada para el equipo multidisciplinar se basa en el concepto de equipo global de desarrollo que exponen Miranda y Sanguino (2007) y que definen como un pequeño número de personas con habilidades complementarias, procedencia funcional y cultural diversa, así como diferente ubicación geográfica, comprometidas en el desarrollo de un nuevo producto y res- ponsables de la gestión de dicho proceso. Si volvemos sobre el gráfico Figura 7 se aprecia claramente este papel del equipo multidisciplinar. Aquí se debe entender, enlazando con el apartado anterior, que el cliente participa en lo que se ha dicho gestión del conocimiento del cliente, diseño del producto, personalización del producto y creación del producto. Todas estas fases que están recogidas en el Círculo del Cliente forman parte fundamental y están integradas en el funcionamiento del equipo multidisciplinar de diseño de producto del esquema que se ha visto en el gráfico Figura 7. Este equipo es el responsable de trasladar hacia los otros elementos de la cadena de suministro todo lo que con el cliente se ha trabajado. Asimismo, el equipo recibe la información del resto de la cadena de suministro para poderlos compartir también con el cliente que forma parte de este equipo multidisciplinar.

De esta manera se garantiza un proceso constante de información en un sentido y en el otro, de forma que el conocimiento que se tiene de los pensamientos y necesidades del cliente está en todo momento presente para así lograr a través de la gestión de la cadena de suministro el producto que el cliente quiere. Todo este flujo de información hacia adentro y hacia afuera del sistema es la interacción que se pretende con el modelo y como dicen Cuenca et al. (2009) los inputs y outputs de los procesos están muy ligadas al sistema empresarial en el que se ejecutan los procesos, así como el sistema de información asociado que da soporte a dicha ejecución Pero no solamente se actúa en la dirección del cliente hacia la cadena, sino que las posibles variaciones por razones técnicas, de constructibilidad, económicas, de seguridad o de cualquier otro tipo que se tengan que realizar dentro del proceso de creación del producto se trasladan de forma inmediata al cliente para su conocimiento. De esta suerte el cliente no descubre nada nuevo cuando se le entrega el producto puesto que ha sido partícipe y conocedor de todo el proceso de creación del producto hasta su final.

El equipo multidisciplinar de diseño de producto es un equipo compuesto por empleados de distintas compañías. Por lo tanto se abre el círculo y se empieza a trabajar inter-compañías en lugar de solamente intra-compañía. Estos dos niveles, el de la compañía y el de la relación de la compañía con sus proveedores se pueden ir variando en función de las actividades que una empresa tenga internamente $y$ aquellas en las que se debe apoyar de forma externa. 
Estas relaciones entre compañías deben llegar a desarrollar alianzas que permitan el funcionamiento eficiente de estos equipos multidisciplinares. Una vez que este equipo ha diseñado el producto y de forma interactiva en todo momento, ya que se está trabajando de forma continua con el cliente, este equipo traslada, en lo que se ha venido a representar con un cuadro exterior, sus conclusiones y trabajos. De forma gráfica se puede entender que el círculo exterior de lo que se ha llamado equipo multidisciplinar de diseño de producto, es como una membrana que mantiene relación constante con todos los elementos que están en el exterior de ese círculo. De esta manera el diseño arquitectónico se comparte con los despachos de ingenieros, despachos de arquitectos, empresas para la construcción, estructuristas, subcontratistas, industriales, etc. Dicha relación tiene un doble sentido, también hacia dentro de la membrana debe fluir información de las empresas externas del círculo. Esta permeabilidad es la que garantiza que en todo momento se esté desarrollando un producto realizable, con las últimas innovaciones, y ajustado a las necesidades del cliente.

Esta integración global no la podemos entender sin una etapa de crecimiento con interacciones individuales dentro del equipo de trabajo, es aquí donde en el modelo presentado aparece el concepto desarrollado para la implantación de la alianza uno a uno de los integrantes de la cadena. El desarrollo de esta alianza en la propuesta del modelo que se presenta se basa en cinco pilares: organización de personas, estrategia institucional, economía de beneficios, técnica e información y cliente. Entendemos que estos cinco pilares son los elementos básicos que garantizan que la alianza tenga un desarrollo a largo plazo y que los objetivos de unión dentro del equipo multidisciplinar de trabajo señalado como núcleo dentro de la propuesta de modelo se cumplan.

Por último, no se debe olvidar que en el mundo en el que se desarrolla el presente modelo, aparece la figura del promotor, que se ha denominado en el presente gráfico con el nombre de: Propiedad. Es la decisión de la Propiedad la que arranca el proceso y la que de forma indirecta supervisa todo su funcionamiento. Con la idea de fidelizar y satisfacer al cliente.

\section{Conclusiones}

A lo largo del artículo hemos visto varios modelos que permiten conceptualizar soluciones al proble- ma de gestión de la Cadena de Suministro en el sector de la construcción. El sector de la construcción está experimentando grandes cambios en todos los aspectos relacionados con su negocio: el mercado, las relaciones entre competidores, los mecanismos de financiación, los recursos de mano de obra, etc. Los modelos que se aportan al sector pretenden crear un concepto de negocio que ayude al desarrollo e implementación de la idea concebida de manera que sea más sencillo y que se obtenga un enfoque adecuado a ciertos problemas detectados en la implantación de los procesos de negocio.

El Modelo de Organización Global presentado tiene dos características que lo distinguen y lo enfocan. La primera es que es un modelo con una participación fundamental del cliente que hace que todos los procesos relacionados con la creación, diseño, construcción del producto, acabados y puesta a disposición estén vinculados con la visión y necesidades que el cliente tiene.

En segundo lugar, otra característica del Modelo de Organización Global presentado es la integración de las cadenas productivas y/o de suministro del sector mediante las relaciones de alianzas entre los distintos elementos de la cadena que permiten entender la organización resultante como un todo dentro del modelo presentado.

En la circunstancias de mercado que nos toca afrontar en la actualidad es importante poder tener modelos de referencia que nos ayuden a conceptualizar procesos de negocio e implantar estrategias diferenciadas haciendo posible el desarrollo de sectores de la actividad comercial de una manera viable. El Modelo de Organización Global será uno de ellos pero deben existir más haciendo posible estrategias distintas de distintos competidores en un mismo sector. Esta riqueza de modelos garantiza un sector dinámico que dé respuesta económica a las necesidades de una sociedad. Es pues necesario el crecimiento de distintos modelos de negocio de forma que aparezcan competidores fuertes en un sector en permanente cambio.

Asimismo, también es bueno, que estos modelos se desarrollen en la práctica y se maticen con las aportaciones de una implantación que acerque el modelo conceptual a la realidad del negocio. Este proceso de acercamiento siempre es un proceso enriquecedor si se concibe como un proceso iterativo en el cual la realidad de la implantación replantea una solución matizada al modelo que la recoge 
y la lanza de nuevo hacia la realidad para su implantación. De esta manera se crea un modelo de riqueza mayor con las aportaciones que vienen de una implantación vivida.

\section{Bibliográfia}

ABUDAYYEH, 0. (1994). «Partnering: a tearn building approach to quality construction management». J. Mgmt. Engrg., ASCE, I0(6), 26-29.

AKINTOYE, A, MCLNTOSH, G AND FITZGERALD, E (2000) «A survey of supply chain collaboration and management in the UK construction industry», European Journalof Purchasing and Supply Management, 6, I59-I 68.

BALLARD, G y HOWELL G A (2003) «Lean project management», Building Research S Information, 31 (2), I 19 133.

BANWELL H.- (1964). «The Placing and Management of Contracts for Building and Civil Engineering Work». HMSO, London.

BOWERBOX, D.J. (1997). «Integrated Supply Chain Management: A Strategic Imperative», presented at the Council of Logistics Management 1997 Annual Conference, 5-8 Oct. Chicago, IL.

BROWN, J. H. (1993). "Partnering on engineering/construction projects." PM Network, 7( I 2), 32-34.

BYGBALLE LENA, MARIANNE JAHRE y ANNA R.S. SVÄRD. (2007). «Partnering in construction - Relationship connectedness»».

CAPÓ VICEDO, JOSEP,EXPÓSITO, LANGA MANUEL y, JOSÉV.TOMÁS MIQUEL (2005). «La importancia de la gestión del conocimiento en la cadena de suministro de la construcción». IX Congreso de Ingeniería de Organización Gijón, 8 y 9 de septiembre de 2005.

CAPÓ VICEDO, JOSEP, EXPÓSITO, LANGA MANUEL y, JOSÉ V.TOMÁS MIQUEL (2009). «Redes virtuales de PYMES. Un caso de estudio en el sector textil español». Revista Dirección y Organización n 38, pags 66-77. ISSN: | 32- I75X.

CRANE,T. G. (1995). "The benefits and implementation of partnering in the construction industry," ME thesis, Clemson Univ., Clemson, S.C.

CRANE,T.G., FELDER, J.P.,THOMPSON, P.J.,THOMPSON, M.G., y SANDERS, B.R., (1997). «Partnering process model». ASCE Journal of Management in Engineering 13 (4), 57-63.

CUENCA, LI., BOZA, A., ALARCÓN, F.y LARIO, F.F. (2009). «Metodología para la identificación de inputs y outputs de proceso de negocio en un entorno colaborativo.»
Revista Dirección y Organización, n³ 37, pags 29-34. ISSN I |32-175X.

EGAN, J. (CONSTRUCTIONTASK FORCE) (1998). «Rethinking Construction», DETR, London.

FAWCETT, S.E. AND MAGNAN, G.M. (2002). «The rhetoric and reality of supply chain integration». International Journal of Physical Distribution \& Logistics Management 32 (5), 339-36I.

GONZALEZ MANUEL, ARRAÑUDA BENITO y FERNÁNDEZ ALBERTO (2000). « Causes of Subcontracting: Evidence from Panel Data on Construction Firms». Journal of Economic Behavior. Citeseer.

HEWITT F. ( 1994). «Supply Chain Redesign». The International Journal of Logistics management, Vol. 5, No. 2, pp. I-9.

LAMMING, R., (1996). «Squaring the lean supply with supply chain management». International Journal of Operations and Production Management 16 (2), I88196.

LATHAM, M., ( 1994). «Constructing the Team». HMSO, London.

LISKA, R., ( 1 993). «Partnering for project success». Assoc. Builders and Contractors. Inc.. Washington, D.C.

LOVE P.E.D. GUNASEKARAN A. y LI, H. , ( I 998), «Putting an engine into re-engineering: toward a process based organisation», International Journal of Operations \& Production Management, Vol. 18 No 9. pp. 937-949.

LOVE P.E.D., SOHAL A.S. (2002). «Influence of organisational learning practices on rework costs in projects». Proceedings of eight international conference on ISO 9000 \& TQM (change Management), Center of Quality Management Research (CMQR) at RMIT University, RMIT Stoney Hall, Melbourne I3-I 5 Abril.

LOVE PETER E. D, ZAHIR IRANI, EDDIE CHENG, HENG LI, (2002) «A model for supporting inter-organizational relations in the supply chain». Engineering Construction and Architectural Management 9 (I), 2-I5.

LOVE P.E.D., ZAHIR IRANI y DAVID J. EDWARDS, (2004), «A seamless supply chain management model for construction». Supply Chain Management: An International Journal Vol. 9 Number I pp 43-56.

MIRANDA GONZALEZ, FRANCISCO JAVIER y SANGUINO GALVAN, RAMÓN (2007). «La gestión de Conocimiento en los equipos globales de desarrollo. Revista Dirección y Organización, n³3, pags 21 -34. ISSN | | 32-175X.

MORRISON, M. y MEZENTSEFF, M. ( 1 997). «Learning alliances, a new dimension of strategic alliances». Management Decision, 35, 35 I-357. 
NARASIMHAN R. y SANTOSH MAHAPATRA. (2004). «Decision models in golbal supply chain management». Industrial Marketing Management 33, 2 1-27.

PEREIRA RAMA ANTONIO y CHAPARRO PELÁEZ JULIÁN (2004). «Un sistema de información Interoganizacional como soporte a la Gestión en el sector de la edificación». ACEDE 2004.

POIRIER. C. C., y HOUSER, W. E ,(1993). «Business partnering for continuous improvement». Berrett-Koehler Publishers, San Francisco, Calf.

PROVERBS, D G. y HOLT, G D (2000) «Reducing construction costs: European best practice supply chain implications». European Journalof Purchasing and Supply Managemenf, 6(Speciai Issue), I49-I58.

REY ROMÁN, ANDRÉS FELIPE y ECHEVERRY, DIEGO (2005). «Prototipo para el control de obras y teorías para el manejo de excesos de liquidez». ICYA 200I060.

SHAOKAI LUA y HONG YANC. (2006). «A model for evaluating the applicability of partnering in construction». International Journal of Project Management. Volume 25, Issue 2, February 2007, Pages I64- I 70. ICCREM 2005, the International Conference on Construction and Real Estate Management 2005.

SCOTT CHARLES y ROY WESTBROOK (199I). «New Strategic Tools for Supply Chain Management». International Journal of Physical Distribution \& Logistics Management Volume: 21 Issue: I Page: 23 - 33. DOI: | 0.1 | 08/09600039| | 0002225 Publisher: MCB UP Ltd.

SIMON REPORT, ( 1 944), «Report of the Management and Planning of Contracts». The Simon Report, HMSO London.

SRIDHAR TAYUR, RAM GANESHAN y MICHAEL MAGAZINE. (1998). «Quantitative Models for Supply Chain Management». Springer. 885 páginas ISBN:0792383443.
STADTLER HARMUNT y KILGER CHRISTOPH. (2008). «Supply Chain Managenent and Advanced Planning: Concepts, Models, Software and Case Studies». Springer. ISBN 978-3-540-745 I I-2.

STEVENS, G.C. (1989). «Integrating the Supply Chain». International Journal of Physical Distribution \& Materials Management 19:3-8.

TOWILL, D. R., NAIM, N.M., y WIKNER, J. ( 1992). «Industrial Dynamics Simulation Models in the Design of Supply Chains». International Journal of Physical Distribution and Logistics Management 22:3- 13.

VÉLEZ MORENO LIGIA MARÍA (200I). «Construcción: Industrialización y Construcción.» http://noticias.arq. com.mx/Detalles/ / 822.html.

VOORDIJK, H, DE HAAN, J y JOOSTEN, G-J (2000) "Changing governance of supply chains in the building industry: a multiple case study». European Journal of Purchasing and supply Management, 6 (Special Issue), 217-225

VRIJHOEF, RUBEN, y LAURI KOSKELA, (2000). «The four roles of supply chain management in construction». European Journal of Purchasing \& Supply Management Volume 6, Issues 3-4, December 2000, Pages $169-178$.

VRIJHOEF, R. y DE RIDDER, H. (2005). «Supply chain integration for achieving best value for construction clients: client-driven versus supplierdriven integration». Proceedings QUT Research Week. 4-6 July 2005, Brisbane.

VRIJHOEF RUBEN. (2007). «Supply Chain Integration in Construction. Design and construction processes». TuDelft Technische Universitiet Delft. Paper series 2.

WINCH, G M (2003) «Models of manufacturing and the construction process: the genesis of re-engineering construction». Building Research \& Informatmn, 3। (2), 107-118. 